ARTICLE

Received 20 Feb 2015 | Accepted 8 Sep 2015 | Published 21 Oct 2015

DOI: $10.1038 /$ ncomms 9577

OPEN

\title{
Photoelectric detection of electron spin resonance of nitrogen-vacancy centres in diamond
}

E. Bourgeois ${ }^{1,2}$, A. Jarmola ${ }^{3}$, P. Siyushev 4 , M. Gulka ${ }^{2,5}$, J. Hruby ${ }^{2,5}$, F. Jelezko ${ }^{4}$, D. Budker ${ }^{6,3}$ \& M. Nesladek ${ }^{1,2}$

The readout of negatively charged nitrogen-vacancy centre electron spins is essential for applications in quantum computation, metrology and sensing. Conventional readout protocols are based on the detection of photons emitted from nitrogen-vacancy centres, a process limited by the efficiency of photon collection. We report on an alternative principle for detecting the magnetic resonance of nitrogen-vacancy centres, allowing the direct photoelectric readout of nitrogen-vacancy centres spin state in an all-diamond device. The photocurrent detection of magnetic resonance scheme is based on the detection of charge carriers promoted to the conduction band of diamond by two-photon ionization of nitrogenvacancy centres. The optical and photoelectric detection of magnetic resonance are compared, by performing both types of measurements simultaneously. The minima detected in the measured photocurrent at resonant microwave frequencies are attributed to the spin-dependent ionization dynamics of nitrogen-vacancy, originating from spin-selective non-radiative transitions to the metastable singlet state.

\footnotetext{
${ }^{1}$ IMOMEC division, IMEC, Wetenschapspark 1, B-3590 Diepenbeek, Belgium. ${ }^{2}$ Institute for Materials Research (IMO), Hasselt University, Wetenschapspark 1, B-3590 Diepenbeek, Belgium. ${ }^{3}$ Department of Physics, University of California, Berkeley, California 94720-7300, USA. ${ }^{4}$ Institute for Quantum Optics and IQST, Ulm University, Albert-Einstein-Allee 11, D-89081 Ulm, Germany. ${ }^{5}$ Czech Technical University in Prague, Sitna sq. 3105,272 01, Kladno, Czech Republic. ${ }^{6}$ Helmholtz Institute, Johannes Gutenberg University, D-55099, Mainz, Germany. Correspondence and requests for materials should be addressed to M.N. (email: milos.nesladek@uhasselt.be).
} 
R eading out the electron spin of the negatively charged nitrogen-vacancy $\left(\mathrm{NV}^{-}\right)$centres in diamond is essential for applications in quantum computing and secure communication $^{1,2}$, as well as for nanoscale magnetic and electric sensing ${ }^{3-5}$ and for non-perturbing sensing and imaging of quantum objects ${ }^{6,7}$. $\mathrm{NV}^{-}$ensembles in bulk diamond are in addition used for high-sensitivity magnetometry, with submicrometre resolution ${ }^{8}$. At present, the readout of $\mathrm{NV}^{-}$centres electron spin state is typically performed optically, by detecting photons using confocal microscopy. No all-diamond readout technique allowing for integration with diamond electronic chips is available. One of the limiting factors for optical detection is the low collection efficiency of photons emitted by $\mathrm{NV}^{-}$centres in bulk diamond, due to the limitations of objective optics and to the high index of refraction of diamond. Reaching an optical detection efficiency higher than a few percents most often requires complex microfabrication ${ }^{9,10}$, even though simpler techniques for the fabrication of diamond photonics structures were recently proposed ${ }^{11}$. Additionally, the use of $\mathrm{NV}^{-}$centres for quantum computing at room temperature is based on the entanglement between the spins of adjacent $\mathrm{NV}^{-}$centres at a distance of the order of $10 \mathrm{~nm}^{12}$. The individual optical readout of such proximal $\mathrm{NV}^{-}$centres requires a resolution below the diffraction limit, which can only be achieved by complex optical techniques such as stimulated emission depletion (STED) microscopy ${ }^{13}$ or reversible saturable optical linear fluorescence transitions (spin-RESOLFT) microscopy 14 .

In the following, we describe an alternative non-optical technique consisting in the direct photoelectric readout of the electron-spin resonance of $\mathrm{NV}^{-}$centres. This scheme is denoted as photocurrent detection of magnetic resonance (PDMR). Similarly to the readout method presented in ref. 15, our detection technique is based on the spin-dependence of $\mathrm{NV}^{-}$ionization dynamics. However, while in ref. 15 the spin detection relies on an optical readout of NV charge state, our method is based on direct electric detection of charge carriers excited from $\mathrm{NV}^{-}$centres to the diamond conduction band, which can be performed electrically on a chip. In contrast to a recently proposed scheme, in which electronic readout of $\mathrm{NV}^{-}$centres spin state is performed by monitoring nonradiative energy transfers to graphene ${ }^{16}$, PDMR detection can be performed in an all-diamond device, without the indirect energy transfer to another material. Photoelectric detection of spin resonances only requires the fabrication of electrodes on the diamond chip by standard lithography. It avoids the complexity of confocal imaging and would allow for example the detection of $\mathrm{NV}^{-}$spin resonance in light-scattering media. We demonstrate the PDMR technique on $\mathrm{NV}^{-}$ensembles; however, it may be developed to be used for the readout of single $\mathrm{NV}^{-}$centres spins. By further downscaling the inter-electrode distance to $10 \mathrm{~nm}$ gaps ${ }^{17,18}$, PDMR has the potential to reach the independent readout of $\mathrm{NV}^{-}$centres situated closer than the diffraction limit. Another peculiar feature of the photocurrent detection principle is the photoelectric gain mechanism ${ }^{19,20}$, which might lead to high detection efficiency since every photon has the ability to generate more than one electron-hole pair.

\section{Results}

Demonstration of PDMR on type-Ib diamond. A schematic representation of the PDMR setup is depicted in Fig. 1a. The principle of PDMR is demonstrated on $\mathrm{NV}^{-}$centres ensembles, by characterizing an irradiated and annealed type-Ib singlecrystal diamond plate of [100] crystallographic orientation (sample E2), containing both $\mathrm{NV}^{-}$centres ( $\sim 20$ p.p.m.) and
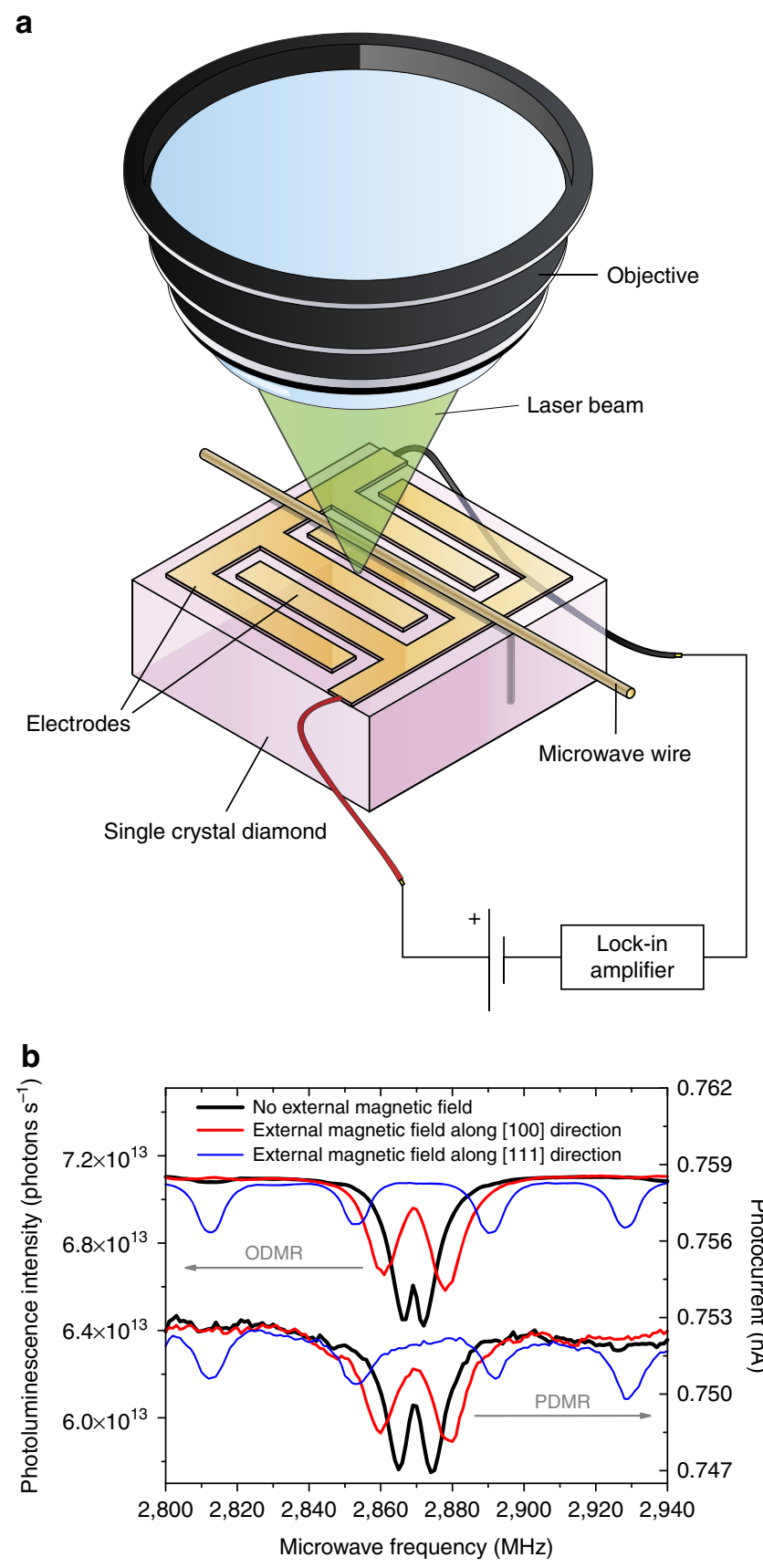

Figure 1 | PDMR on NV centres in type-Ib diamond. (a) Schematic representation of the setup used for PDMR. (b) Comparison of ODMR and PDMR spectra recorded simultaneously. Sample E2, green light power: $100 \mathrm{~mW}$, applied electric field: $5 \times 10^{4} \mathrm{~V} \mathrm{~cm}^{-1}$, light pulse duration: $940 \mu \mathrm{s}$, distance between contacts: $100 \mu \mathrm{m}$, magnitude of the external magnetic field: $0.50 \mathrm{mT}$ along the [100] direction, $2.0 \mathrm{mT}$ along the [111] direction.

substitutional nitrogen centres $\left(\mathrm{N}_{\mathrm{s}}^{0}, \sim 200\right.$ p.p.m. $)$. Optical detection of magnetic resonance (ODMR) and PDMR were performed simultaneously on this sample. For this, the intensity of photoluminescence and the photocurrent were measured simultaneously while scanning the microwave frequency in the absence and in the presence of an external magnetic field (Fig. 1b). Minima in the photoluminescence intensity are observed at microwave frequencies inducing resonant transitions between the $\mid 0>$ and the $\mid \pm 1>$ spin sublevels of the $\mathrm{NV}^{-}$spin triplet ground state $\left({ }^{3} \mathrm{~A}_{2}\right)^{21}$. Minima in photocurrent are clearly detected at identical frequencies, demonstrating that 
photocurrent measurements can be used to detect the spin resonances of $\mathrm{NV}^{-}$centres.

The two resonances observed in ODMR and PDMR spectra in the absence of an external magnetic field indicate the existence of a splitting between linear combinations of the $\mid+1>$ and $\mid-1>$ spin sublevels of $\mathrm{NV}^{-}$(hereafter referred to as ' $\mid \pm 1>$ spin manifold'), induced by local strain in the material ${ }^{8}$ and, in the case of PDMR measurements, by the externally applied electric field. We will refer to this splitting between magnetic resonances at zero-magnetic field as 'zero-field splitting' (ZFS). The origin of the difference between the ZFS observed in ODMR and PDMR is discussed in the Supplementary Note 1. In the presence of an external static magnetic field applied using a permanent magnet, a further splitting of resonant frequencies is observed both in ODMR and PDMR spectra, reflecting the perturbation of the $\mid \pm 1>$ spin manifold of $\mathrm{NV}^{-}$ground state because of the Zeeman effect. As expected ${ }^{22}$, two magnetic resonances are observed when the magnetic field is applied along the [100] direction of the diamond crystal and four when it is applied along the [111] direction (Fig. 1b).

Investigation of PDMR mechanism. To investigate the photoionization mechanism inducing the photocurrent minima at $\mathrm{NV}^{-}$spin-resonance frequencies, the photocurrent detected on sample E2 was measured as a function of the green light power (Fig. 2). A good fit to the experimental data is obtained using the sum of a linear and a quadratic function, indicating that the measured photocurrent results from the combination of a one-photon and a two-photon ionization processes ${ }^{23}$. In previous experiments $^{23}$ and in theoretical studies ${ }^{24,25}$ it was demonstrated that a photon energy higher than $2.6 \mathrm{eV}$ is necessary to induce the photoionization of $\mathrm{NV}^{-}$via a one-photon process, that is, to directly promote an electron from the ground state of $\mathrm{NV}^{-}$to the conduction band. Based on this argument and on the quadratic power dependence of the photocurrent, we conclude that a two-photon absorption process is responsible for the $\mathrm{NV}^{-}$-related part of the photocurrent induced by green light $(2.33 \mathrm{eV})$. The mechanism for the two-photon ionization of $\mathrm{NV}^{-}$centres in diamond has been experimentally established $^{23,26,27}$ and modelled ${ }^{27}$. In this process, the absorption of a first photon promotes an electron from the ${ }^{3} \mathrm{~A}_{2}$ triplet ground

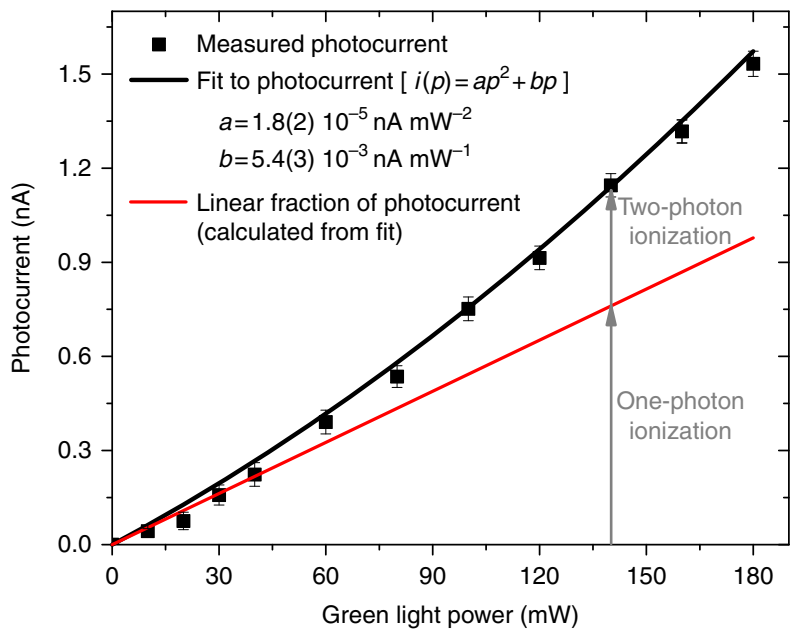

Figure 2 | Light power dependence of photocurrent in type-lb diamond. Sample E2, applied electric field: $5 \times 10^{4} \mathrm{~V} \mathrm{~cm}^{-1}$, light pulse duration: $940 \mu \mathrm{s}$, distance between contacts: $100 \mu \mathrm{m}$. i: photocurrent, $p$ : optical power, $a$ and $b$ : fitting prefactors. Error bars represent the s.d. from the mean of 32 photocurrent measurements. state of $\mathrm{NV}^{-}$to its ${ }^{3} \mathrm{E}$ triplet excited state (Fig. 3a, transition (1)) and a second photon excites this electron to the conduction band of diamond (Fig. 3b, (4)), which results in the conversion of the $\mathrm{NV}$ centre to its neutral state $\mathrm{NV}^{0}$ and in the promotion of an electron into the conduction band (Fig. 3c). To ensure the charge neutrality in the PDMR detection circuit, the $\mathrm{NV}^{0}$ centres formed by ionisation of $\mathrm{NV}^{-}$have to be subsequently converted back to the $\mathrm{NV}^{-}$state (Fig. 3d), either by capturing an electron from a donor defect (in particular from $\mathrm{N}_{\mathrm{s}}^{0}$, present in high concentration in the type-Ib sample under study) ${ }^{28,29}$ or by two-photon conversion from $\mathrm{NV}^{0}$ to $\mathrm{NV}^{-}$. In the latter process, the absorption of a first photon excites the $\mathrm{NV}^{0}$ centre (Fig. 3c, (5)), while a second photon promotes an electron from the valence band to the vacated orbital of $\mathrm{NV}^{0}$ (Fig. 3c, (6)), leaving a hole in the valence band ${ }^{23,27}$. This photo-induced process does not require the presence of electron-donor defects in the diamond crystal.

The linear contribution to the measured photocurrent (Fig. 2) is likely associated with one-photon ionization of $\mathrm{N}_{\mathrm{S}}^{0}$. $\mathrm{N}_{\mathrm{S}}^{0}$ is indeed the dominant point defect in the E2 sample, and the threshold energy for the photoionization of this defect ${ }^{30,31}$ is well below the $2.33 \mathrm{eV}$ excitation energy used in our experiment.

The mechanism proposed to explain the photoelectric detection of $\mathrm{NV}^{-}$magnetic resonances is presented in Fig. 3. The minima observed in the photocurrent at resonant microwave frequencies inducing transitions from the $\mid 0>$ to the $\mid \pm 1>$ electron spin sublevels of $\mathrm{NV}^{-}$ground state $\left({ }^{3} \mathrm{~A}_{2}\right)$ indicate that the photoionization dynamics of $\mathrm{NV}^{-}$is spin-dependant. In ODMR, electrons are initially pumped into the ${ }^{3} \mathrm{~A}_{2} \mid 0>$ spin state under the effect of green illumination, from which they are coherently driven to the ${ }^{3} \mathrm{~A}_{2} \mid \pm 1>$ spin sublevels by the resonant microwave field ${ }^{32}$. Photoluminescence originates from the radiative decay of electrons from the excited state $\left({ }^{3} \mathrm{E}\right)$ to the ground state (Fig. 3b, (2)). Because of spin-selective intersystem crossing ${ }^{33}$, electrons in the ${ }^{3} \mathrm{E} \mid \pm 1>$ sublevels can decay nonradiatively to the ${ }^{1} \mathrm{~A}_{1}$ singlet state (lifetime $\leq 1 \mathrm{~ns}$ ) ${ }^{34}$ from which they further fall into the ${ }^{1} \mathrm{E}$ metastable singlet state (electron shelving). This non-radiative decay path (Fig. $3 \mathrm{~b},(3)$ ) induces a difference between the brightness of the transitions associated with the different $\mathrm{NV}^{-}$spin sublevels, which provides the contrast monitored by ODMR. For PDMR, we expect that electron shelving is also the dominant mechanism explaining the observed contrast. Electrons excited to the ${ }^{3} \mathrm{E} \mid \pm 1>$ sublevels have a non-zero probability to undergo the shelving transitions via the ${ }^{1} \mathrm{~A}_{1}$ and ${ }^{1} \mathrm{E}$ states. For the time during which an electron initially in the ${ }^{3} \mathrm{~A}_{2} \mid \pm 1>$ state undergoes these non-radiative transitions, it does not contribute to the photocurrent. Specifically, the ${ }^{1} \mathrm{E}$ metastable state has a lifetime of $220 \mathrm{~ns}$ at room temperature ${ }^{34}$. For that period, the ${ }^{1} \mathrm{E}$ state stores the electron which leads to a temporary decrease in the occupation of $\mathrm{NV}^{-}$ground state and reduces the rate of two-photon ionization (proportional to the occupation of ${ }^{3} \mathrm{~A}_{2}$ ). This process probably induces the observed magnetic resonances in photocurrent. A similar mechanism was proposed recently to explain the spindependence of $\mathrm{NV}^{-}$ionization dynamics ${ }^{15}$.

An alternative photoionization path would be the direct promotion of electrons from the ${ }^{1} \mathrm{E}$ metastable state to the conduction band. This ionization path would lead to a positive sign of PDMR, since electrons in the ${ }^{3} \mathrm{E} \mid \pm 1>$ sublevels have a higher probability to decay non-radiatively to the singlet state, which leads to a higher occupation of the ${ }^{1} \mathrm{E}$ metastable state. However, the negative resonances observed in PDMR spectra (Fig. 1b) suggest that the contribution of this photoionization process to the total photocurrent at the resonant microwave frequency is significantly lower than the contribution of direct transitions from the excited state ${ }^{3} \mathrm{E}$ to the conduction band. 

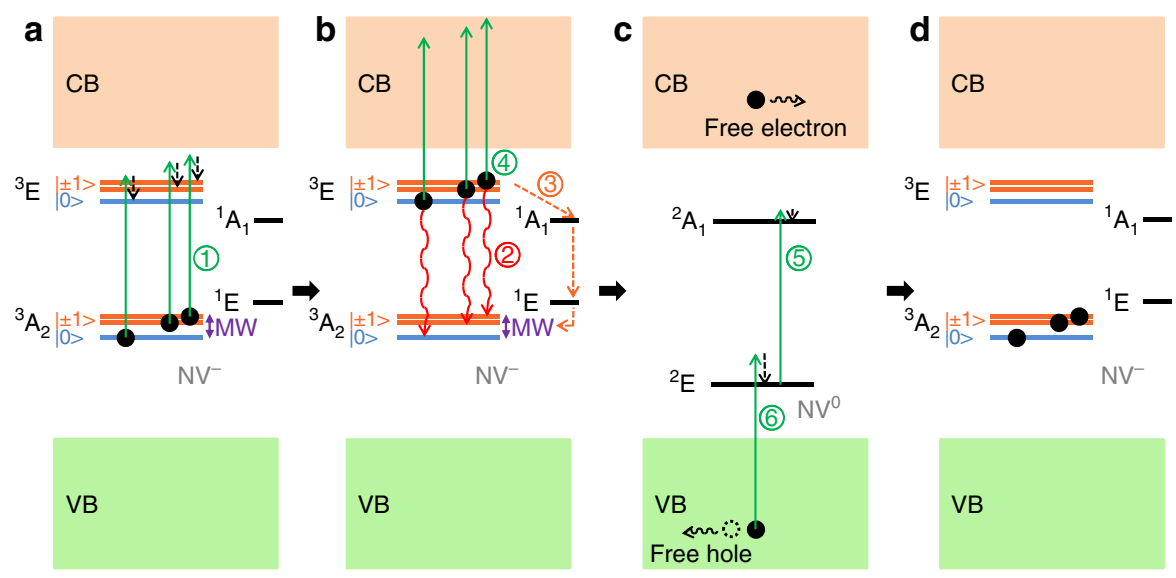

Figure 3 | Mechanism proposed to explain PDMR measurements. A simplified electronic energy level scheme (not to scale) of the NV ${ }^{-}$centre at room temperature is represented (MW: microwave). (a) The absorption of a first photon promotes an electron from the ${ }^{3} \mathrm{~A}_{2}$ ground state to the ${ }^{3} \mathrm{E}$ state of $\mathrm{NV}^{-}$(transition (1)). (b) From ${ }^{3} \mathrm{E}$, electrons can radiatively decay back to ${ }^{3} \mathrm{~A}_{2}$ (transition (2)) or be excited to the diamond conduction band (CB) by the absorption of a second photon (transition (4)). The spin-selective non-radiative decay of electrons to the singlet state ${ }^{1} A_{1}$ (transition (3)), followed by transition to the metastable state ${ }^{1} \mathrm{E}$, enables PDMR and ODMR. (c) The two-photon ionization of $\mathrm{NV}^{-}$results in the formation of a $\mathrm{NV}^{0}$ centre and a free electron in the CB. By a two-photon process in which the $\mathrm{NV}^{0}$ centre is first excited (transition (5)) and an electron is then promoted from the valence band $(V B)$ to the vacated orbital of $\mathrm{NV}^{0}$ (transition (6)), the NV centre can finally be converted back to its negatively charged state (d).

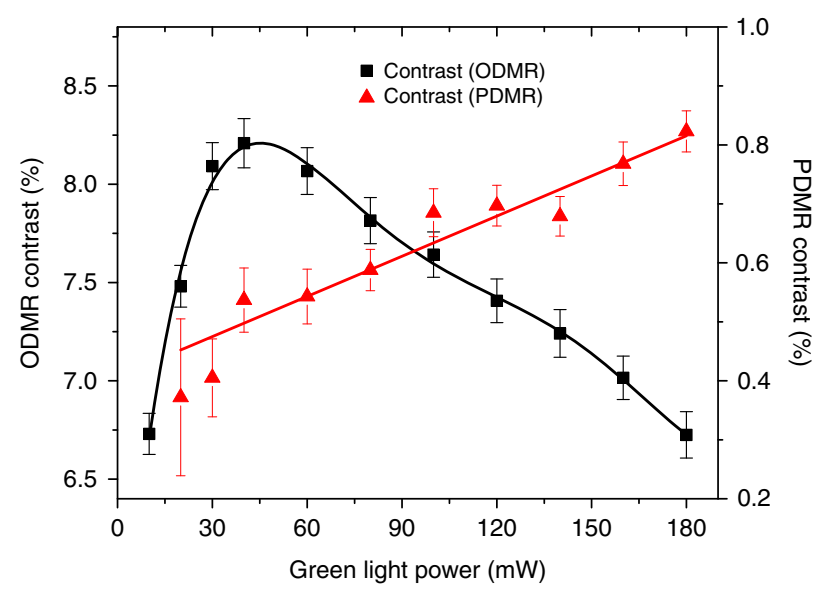

Figure 4 | Light power dependence of PDMR and ODMR constrasts. Sample E2, applied electric field: $5 \times 10^{4} \mathrm{Vcm}^{-1}$, light pulse duration: $940 \mu \mathrm{s}$, distance between contacts: $100 \mu \mathrm{m}$. Error bars represent the s.e.m. of the fitted values (see Methods for details on the fitting procedure). Solid lines are guides for the eye.

PDMR contrast. Figure 4 depicts the light power dependence of the ODMR and PDMR contrasts measured on sample E2. The maximal observed contrasts are $\sim 0.8 \%$ for PDMR and $8.2 \%$ for ODMR. This is partly due to the fact that only the two-photon fraction of the photocurrent (associated with the ionization of $\mathrm{NV}^{-}$) gives rise to detectable electron spin resonances, while its linear fraction is not affected by the microwaves. In ODMR, the contrast is observed to decrease for green light powers above $45 \mathrm{~mW}$, which can be explained by the fact that at strong light power the electron spin polarisation rate becomes larger that the Rabi frequency of the microwave driving field ${ }^{35}$. In the case of PDMR measurements performed on type-Ib diamond, a monotonous increase of the PDMR contrast with the light intensity is observed (Fig. 4). The absence of saturation detected in the PDMR contrast can be explained by the fact that increasing the light power leads to an increase in the quadratic fraction of the photocurrent (associated with ionization of $\mathrm{NV}^{-}$centres) with respect to the linear fraction (associated with ionization of $\mathrm{N}_{\mathrm{S}}^{0}$ ). However, while the quadratic fraction of the photocurrent increases by a factor four between 30 and $180 \mathrm{~mW}$ (Fig. 2), the PDMR contrast only increases by a factor of two, which seems to reflect the fact that as the optical power increases, the spin polarization rate becomes higher than the Rabi frequency, as discussed above. For a quantitative evaluation of this phenomenon, the rate of shelving transitions to the ${ }^{1} \mathrm{E}$ singlet state and the two-photon ionization rate have to be further investigated. The comparison between the light powerdependence of the linewidths of ODMR and PDMR resonances is presented in Supplementary Fig. 1 and commented in Supplementary Note 2.

Influence of the applied electric field. Since the photoelectric detection technique requires the application of a DC electric field to drive the generated charge carriers toward electrodes, the influence of the magnitude of the applied electric field on PDMR spectra was studied. The presence of an external electric field affects the electronic spin sublevels of $\mathrm{NV}^{-}$centres ground state, due to the Stark effect ${ }^{36-38}$. Normalized ODMR and PDMR spectra recorded under different electric fields on sample E2 are presented in Supplementary Fig. 2. No measurable effect of the external electric field on the ODMR signal is detected, even under high applied electric field $\left(5 \times 10^{4} \mathrm{~V} \mathrm{~cm}^{-1}\right)$. Concerning PDMR measurements, it can be observed that in spite of a decrease in the photocurrent, magnetic resonances are clearly detected under an applied electric field as low as $1.3 \times 10^{2} \mathrm{~V} \mathrm{~cm}^{-1}$, corresponding to a local electric field of $3.3 \times 10^{2} \mathrm{~V} \mathrm{~cm}^{-1}$ in diamond. Considering the axial and transverse electric susceptibility parameters of 0.35 and $17 \mathrm{Hzcm} \mathrm{V}^{-1}$, respectively, reported in ref. 36, this local electric field applied along the axis of an $\mathrm{NV}^{-}$centre leads to a splitting at zero-magnetic field of $5.7 \mathrm{kHz}$ between the two proper eigenstates in the $\mid \pm 1>$ manifold and to a shift of $120 \mathrm{~Hz}$ of the splitting between the $\mid 0>$ and the $\mid \pm 1>$ spin sublevels ${ }^{38}$, which is orders of magnitude lower than the full-width at half-maximum (FWHM) of the detected resonances. The impact of the applied electric field on PDMR linewidth and ZFS is presented in Supplementary Fig. 3a,b, and discussed in Supplementary Note 1.

PDMR on type-IIa diamond with low $\mathrm{NV}^{-}$concentration. To demonstrate the possibility of performing PDMR with $\mathrm{NV}^{-}$ ensembles in type-IIa diamond with low $\mathrm{N}_{S}^{0}$ concentration, we 
have used an electron-irradiated and annealed optical grade diamond with estimated $\mathrm{N}_{\mathrm{S}}^{0}$ concentration below 1 p.p.m. and $\mathrm{NV}^{-}$ concentration around 10 p.p.b. (sample E7). We were able to detect the $\mathrm{NV}^{-}$spin electron resonance in the photocurrent measured on this sample (PDMR spectrum compared with ODMR spectrum in Fig. 5a). The light power-dependence of the photocurrent detected on this sample is presented in Fig. 5b. The experimental data can be fitted with the sum of a quadratic function (corresponding to a two-photon ionization process) and a linear function (corresponding to a one-photon ionization process). By comparing with the photocurrent measured on typeIb diamond (Fig. 2), it appears that the ratio between the linear and quadratic prefactors $b$ and $a$ (see Fig. 5b) is about 300 times lower for type-IIa diamond than for type-Ib diamond. This suggests that in the volume of sample E7 contributing to the photocurrent, the concentration of $\mathrm{N}_{\mathrm{s}}^{0}$ defects is low and that a nearly pure two-photon ionization process occurs, without major contribution from the ionization of $\mathrm{N}_{S}^{0}$ defects to the photocurrent. Correspondingly, a higher PDMR contrast is obtained on type-IIa than on type-Ib diamond. Indeed, a PDMR contrast of 3\% was observed on type-IIa diamond in optimal conditions (corresponding to $3 \%$ of the laser power and $0.8 \%$ of the microwave power leading to optimal contrast on type-Ib diamond). This result shows that, as expected, the PDMR technique is more efficient on type-IIa diamond than on type-Ib diamond, due to the
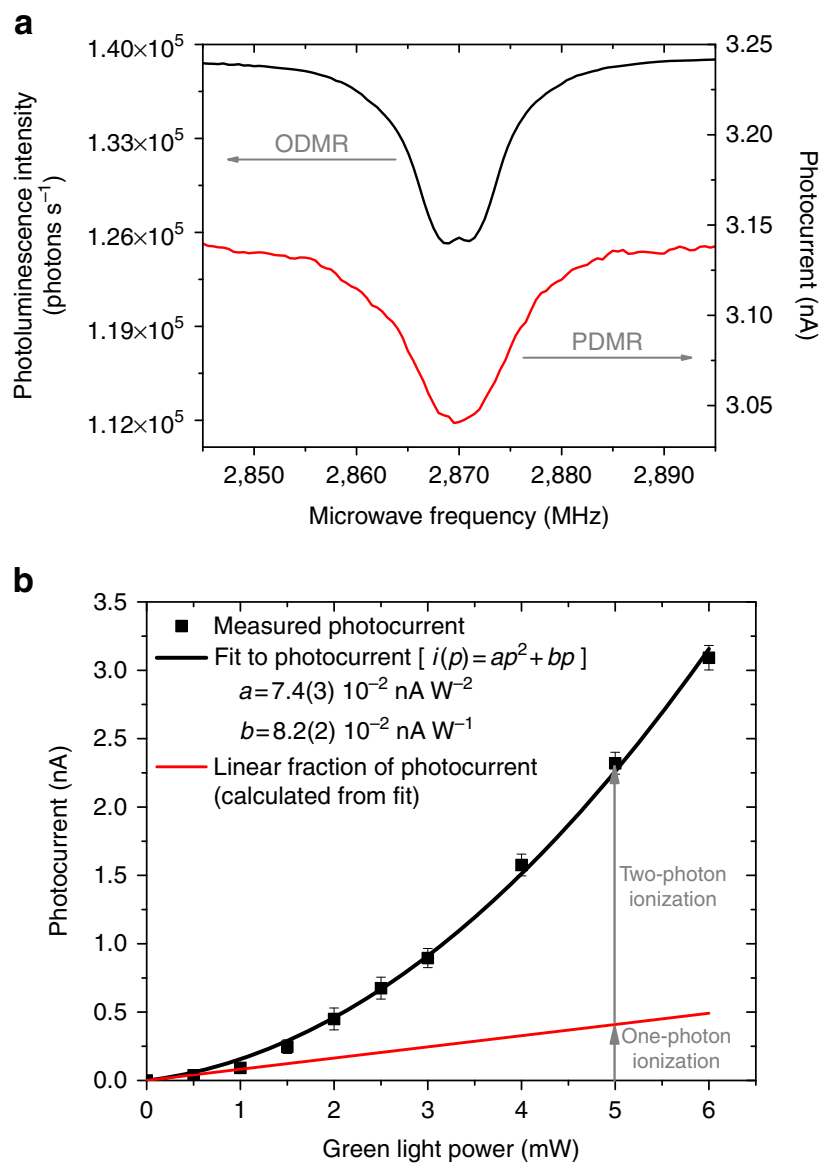

Figure 5 | PDMR on NV centres in type-Ila diamond. Sample E7, light pulse duration: $940 \mu \mathrm{s}$, applied electric field: $3.3 \times 10^{4} \mathrm{~V} \mathrm{~cm}^{-1}$, distance between contacts: $15 \mu \mathrm{m}$. (a) Comparison of ODMR and PDMR spectra measured simultaneously in the absence of external magnetic field (green light power: $6 \mathrm{~mW}$ ). (b) Photocurrent as a function of the $532 \mathrm{~nm}$ light power. $i$ : photocurrent, $p$ : optical power, $a$ and $b$ : fitting prefactors. Error bars represent the s.d. from the mean of measured values. lower contribution of $\mathrm{N}_{\mathrm{S}}^{0}$ photoionization to the total photocurrent.

PDMR sensitivity. To assess the sensitivity of PDMR detection and its potential use for the characterization of single $\mathrm{NV}^{-}$ centres, the efficiency $\eta$ of charge carrier creation was estimated. In a first approximation, the magnitude of the electric field is considered as uniform in the diamond crystal and the field lines are considered as half ellipses extending between electrodes. The photocurrent associated with the two-photon ionization of $\mathrm{NV}^{-}$ centres (quadratic fraction of the photocurrent) $i_{\mathrm{NV}}$ can be expressed as

$$
i_{\mathrm{NV}}=\frac{e \gamma \eta N_{\mathrm{p}}^{2} n_{\mathrm{NV}} V_{1}}{\Gamma}\left(1-\frac{2 V_{2}}{\tau \mu S F_{\mathrm{app}}}\right),
$$

where $e$ is the elementary charge (C), $\gamma$ the photoelectric gain, $N_{\mathrm{p}}$ is the number of incident photons per second $\left(s^{-1}\right), n_{\mathrm{NV}}$ the density of $\mathrm{NV}^{-}$centres in the sample $\left(\mathrm{cm}^{-3}\right), V_{1}$ the volume containing the $\mathrm{NV}^{-}$centres contributing to the photocurrent $\left(\mathrm{cm}^{3}\right), \Gamma$ the decay rate from $\mathrm{NV}^{-}$excited state to $\mathrm{NV}^{-}$ground state $\left(s^{-1}\right), V_{2}$ the volume in which the drift of free charge carriers takes place $\left(\mathrm{cm}^{3}\right), \tau$ the recombination lifetime of charge carriers (s), $\mu$ the electron mobility $\left(\mathrm{cm}^{2} \mathrm{~V}^{-1} \mathrm{~s}^{-1}\right), S$ the axial cross section of the generation volume $V_{1}\left(\mathrm{~cm}^{2}\right)$ and $F_{\text {app }}$ is the applied electric field $\left(\mathrm{V} \mathrm{cm}^{-1}\right)$. The derivation of this expression and the evaluation of the different parameters contained in the formula are detailed in the Supplementary Note 3.

It should be noted that contrary to photoluminescence, the photoelectron generation will not saturate at high pumping power, since the ${ }^{3} \mathrm{~A}_{2} \leftrightarrow{ }^{3} \mathrm{E}$ transition and the subsequent transition from ${ }^{3} \mathrm{E}$ to the conduction band do not saturate. The photocurrent is therefore only limited by the reformation of $\mathrm{NV}^{-}$centres from $\mathrm{NV}^{0}$ centres by re-pumping electrons from the valence band.

The gain $\gamma$ of a photoconductor detector with ohmic contacts is defined as the number of charges collected at electrodes for each photogenerated charge carrier ${ }^{19}$. If the lifetime of one of the charge carriers is longer than its transit time, it will be able to transit several times in between electrodes before recombining. During this time, the boundary conditions (continuity of the current) for ohmic electrodes force one of the electrodes to provide charge carriers of the opposite polarity. In case of charge carriers induced by the photoionization of $\mathrm{NV}^{-}$centres, the process continues as long as the hole remains localized on the NV centre. The photoelectric gain $\gamma$ is therefore equal to the ratio between the recombination lifetime $\tau$ and the electron transit time $t_{\text {transit }}{ }^{19}$. The transit time $t_{\text {transit }}(\mathrm{s})$ can be expressed as a function of the electron mobility $\mu\left(\mathrm{cm}^{2} \mathrm{~V}^{-1} \mathrm{~s}^{-1}\right)$, the distance $L$ between electrodes $(\mathrm{cm})$ and the voltage $U$ applied between electrodes $(\mathrm{V})$ :

$$
t_{\text {transit }}=\frac{L^{2}}{\mu U}
$$

which leads to the following expression for the photoelectric gain:

$$
\gamma=\frac{\tau \mu U}{L^{2}}
$$

For the type-Ib diamond (sample E2), we assume a charge carrier mobility ${ }^{39}$ of $3.6 \times 10^{2} \mathrm{~cm}^{2} \mathrm{~V}^{-1} \mathrm{~s}^{-1}$ and a lifetime between $80 \mathrm{ps}$ and $3 \mathrm{~ns}^{40}$. Based on the electron mobility and lifetime reported for ultra-high purity type-IIa diamond ${ }^{41}$, we assume a charge carrier mobility between $4.5 \times 10^{2}$ and $4.5 \times 10^{3} \mathrm{~cm}^{2} \mathrm{~V}^{-1} \mathrm{~s}^{-1}$ and a charge carrier lifetime between $100 \mathrm{~ns}$ and $2 \mu$ s for the irradiated and annealed optical grade type-IIa diamond under study (sample E7). Considering these ranges of mobility and lifetime, we estimate a photoelectric gain between 1 and 40 for 
measurements performed on type-Ib diamond and between $1 \times 10^{3}$ and $2 \times 10^{5}$ for type-IIa diamond. The higher photocurrent detected per $\mathrm{NV}^{-}$centre for type-IIa diamond $\left(1 \times 10^{-15} \mathrm{~A}^{-1} \mathrm{NV}^{-}\right.$centre under $6 \mathrm{~mW}$ illumination $)$ than for type-Ib diamond $\left(2 \times 10^{-19} \mathrm{~A}^{-1}\right.$ per $\mathrm{NV}^{-}$centre under $100 \mathrm{~mW}$ illumination) results therefore partly from the higher photoelectric gain.

Equation (1) has been used to estimate the efficiency of charge generation $\eta$, based on the quadratic fraction of the photocurrent measured with contacts separated by $15 \mu \mathrm{m}$ and considering the ranges of charge mobility and charge carrier lifetime mentioned above. In the case of a single $\mathrm{NV}^{-}$centre implanted in electronic grade CVD diamond, a very high photoelectric gain can theoretically be obtained, due to the higher lifetime (up to $2 \mu \mathrm{s}$ ) and mobility (up to $4500 \mathrm{~cm}^{2} \mathrm{~V}^{-1} \mathrm{~s}^{-1}$ ) of charge carriers ${ }^{41}$. Assuming the mobility and lifetime mentioned above, a distance of $10 \mathrm{~nm}$ between electrodes ${ }^{17,18}$ and an applied electric field of $5 \times 10^{4} \mathrm{~V} \mathrm{~cm}^{-1}$, a gain of $5 \times 10^{8}$ can theoretically be expected. Considering the efficiency of charge creation $\eta$ determined on the type-IIa diamond under study and a photoelectric gain of $5 \times 10^{8}$, the two-photon ionization of a single $\mathrm{NV}^{-}$centre illuminated at $I_{\text {sat }}$ (light intensity necessary to saturate the ${ }^{1} \mathrm{E}$ singlet state, estimated $^{42}$ to be $\sim 600 \mathrm{MW} \mathrm{m}^{-2}$ ) would induce a photocurrent between $6 \mathrm{fA}$ and $1 \mathrm{pA}$, which is measurable, for example by lockin amplification. Our calculation indicates therefore that PDMR could potentially be used for the characterization of single $\mathrm{NV}^{-}$ centres in electronic grade type-IIa diamond.

PDMR bandwidth. To investigate the bandwidth of the photoelectric detection technique and to demonstraste the possibility to employ it for pulsed spin detection experiments (Ramsey or Rabi oscillations, spin-echo experiments and so on), faster photocurrent measurements were performed on the E2 sample (see Supplementary Note 4). The photoelectric detection of spin magnetic resonances was achieved using light pulses down to $10 \mu \mathrm{s}$, with lock-in amplification of the photocurrent. No significant effect of the reduction of light pulse duration on the linewidth and contrast of photocurrent resonances was observed (Supplementary Fig. 4a). The detection of the photocurrent induced by light pulses as short as $50 \mathrm{~ns}$ was in addition achieved using a charge sensitive preamplifier instead of the lock-in technique (Supplementary Fig. $4 \mathrm{~b}$ and Supplementary Fig. 5a and b). This demonstrates the possibility to perform a fast readout of the photocurrent and shows that the photoelectric detection method may be used for pulsed spin-resonance experiments, for which readout laser pulses between 200 and $500 \mathrm{~ns}$ are typically used $^{43,44}$.

In conclusion, we have demonstrated a new principle for the readout of $\mathrm{NV}^{-}$centres spin magnetic resonances in diamond. By reducing the contact area, this technique has the potential to address single $\mathrm{NV}^{-}$centres. This paradigm may lead to a sensitive way for the construction of diamond nanoscale sensors and quantum devices and their readout, allowing directly performing quantum operation on a chip.

\footnotetext{
Methods

Experimental setup. The samples characterized by ODMR and PDMR were prepared by electron-irradiating a high-pressure high-temperature type-Ib single-crystal diamond $\left(2.9 \times 2.9 \times 0.5 \mathrm{~mm}^{3}\right.$ plate from Element Six $)$ with an initial concentration of approximately 200 p.p.m. of $\mathrm{N}_{\mathrm{s}}^{0}$ (sample E2) and a chemical vapour deposited optical grade type-IIa single-crystal diamond $(2.8 \times 2.8 \times 0.28$ $\mathrm{mm}^{3}$ plate from Element Six) with an initial concentration of $\mathrm{N}_{\mathrm{S}}^{0}$ below 1 p.p.m. (sample E7). Electron irradiation at $14 \mathrm{MeV}$ with doses of $10^{18}$ and $10^{16} \mathrm{~cm}^{-2}$ for type-Ib and type-IIa samples, respectively, was performed at the Mainz Microtron. After electron irradiation, the samples were annealed for $4 \mathrm{~h}$ at $700{ }^{\circ} \mathrm{C}$ in vacuum, leading to a concentration of $\mathrm{NV}^{-}$centres of $\sim 20$ p.p.m. in E2 and $\sim 10$ p.p.b. in
} E7. Before the fabrication of electrodes, the diamond crystals were cleaned and oxidized for $30 \mathrm{~min}$ in an acidic mixture of $\mathrm{H}_{2} \mathrm{SO}_{4}$ and $\mathrm{KNO}_{3}$ at $250^{\circ} \mathrm{C}$, and rinsed with ultrapure water. Coplanar interdigitated electrodes with a distance of 15 or $100 \mu \mathrm{m}$ were then prepared on one of the faces of the diamond crystals by lift-off photolithography and sputtering of $20 \mathrm{~nm}$ of titanium topped by $80 \mathrm{~nm}$ of aluminium. After metal sputtering, the samples were annealed in vacuum at $510^{\circ} \mathrm{C}$ for $3 \mathrm{~h}$ to form Ohmic contact between diamond and electrodes.

For ODMR and PDMR measurements, the $532 \mathrm{~nm}$ illumination is produced by a linearly polarized single-mode Nd:YAG Laser (Gem 532 from Quantum Laser).

For measurements on sample E2, this light is pulsed by an acousto-optical modulator (AOM; model 3200-146 from Crystal Technology) with a diffraction efficiency of $80 \%$ and a contrast ratio of 1,000:1. Except for the study of the influence of light pulse duration on ODMR and PDMR signal, all the measurements were performed with a pulsing frequency of $531 \mathrm{~Hz}$. The pulsed light beam is focused onto the diamond surface using a $\times 10$ air objective with a numerical aperture of 0.28 and a working distance of $33.5 \mathrm{~mm}$, resulting in a light spot with a diameter around $7 \mu \mathrm{m}$. This $7 \mu \mathrm{m}$ light spot is positioned in the 15 or $100 \mu \mathrm{m}$ gap separating the coplanar electrodes. Photoluminescence is measured in a non-confocal configuration. Photoluminescence light is collected by the same objective and filtered using a dichroic mirror with a cut-on wavelength of $552 \mathrm{~nm}$ and a sharp-edge long-pass filter with a cut-on wavelength of $550 \mathrm{~mm}$ (optical density $>5$ in the rejection region). The photoluminescence light is focused onto a pyroelectric detector. The output voltage of the pyroelectric detector is measured with a lock-in amplifier (7260 DSP from EG\&G), referenced to the AOM pulsing (time constant: $50 \mathrm{~ms}$ ).

For measurements on sample E7, the $532 \mathrm{~nm}$ light is pulsed at a frequency of $531 \mathrm{~Hz}$ using a chopper wheel. The pulsed light beam is focused onto the diamond surface using an air objective with a numerical aperture of 0.82 and a working distance of $400 \mu \mathrm{m}$, resulting in a light spot with a diameter $\sim 0.8 \mu \mathrm{m}$. This light spot is positioned in the $15 \mu \mathrm{m}$ gap separating the coplanar electrodes. The photoluminescence light is measured by an avalanche photodiode.

For photocurrent measurements on samples E2 and E7, the photo-carriers generated upon green illumination are driven towards electrodes by applying a DC voltage between coplanar contacts. For light pulses between 10 and $940 \mu$ s, the photocurrent is amplified by a low-noise current to voltage preamplifier (SR570 from SRS, gain: $20 \mathrm{nA} \mathrm{V}^{-1}$ ), and measured using a lock-in amplifier (SR830 from SRS) referenced to the AOM or chopper wheel frequency (time constant: $30 \mathrm{~ms}$ ). The settings of the lock-in amplifier are chosen so that the magnitude of the signal vector (which does not depend on the phase between the signal and lock-in reference) is recorded. We measure therefore the photocurrent intensity, independent of the sign of the photocurrent. Lock-in amplification leads to high signal-to-noise ratio. The photocurrent measured in our experiments was in the range of $10 \mathrm{pA}$ to $1 \mathrm{nA}$ and was detected with a four-digit precision. For fast photocurrent measurements (light pulses between 50 and $400 \mathrm{~ns}$ ), the photocurrent is amplified using a charge sensitive preamplifier with a rise time of $2.5 \mathrm{~ns}$ and a sensitivity of $4 \mathrm{~V} / \mathrm{pC}$ (A250CF CoolFET from AMPTEK INC.). The high sensitivity of this amplifier allows the detection of 48 electrons in a single pulse. The output signal of the preamplifier is measured using a digital real time oscilloscope (TDS 620B from Tektronix, bandwidth: $500 \mathrm{MHz}$ ). The DC offset of the charge sensitive preamplifier was subtracted from the measured signal to obtain the data presented in Supplementary Figs $4 \mathrm{~b}$ and 5a,b. The presented signals were in addition smoothed to remove high-frequency noise.

Microwaves of controlled frequency are produced with a $200-4,000 \mathrm{MHz}$ radiofrequency signal generator (TEG4000-1 from Telemakus) with an output power of $1 \mathrm{~mW}$. The microwave power is set to $40 \mathrm{~mW}$ (Fig. 5a), 1W (Supplementary Figs 2 and $3 \mathrm{a}, \mathrm{b}$ ) or $5 \mathrm{~W}$ (Figs $1 \mathrm{~b}$ and 4, Supplementary Figs 1, 4a and 6a,b) using an attenuator (TEA4000-3 from Telemakus) and a broadband amplifier with a gain of $45 \mathrm{~dB}$ (ZHL-16W-43 + from Mini-circuits). The microwave field is applied using a metal wire pressed across the diamond surface and connected to a $50 \Omega$ terminator. The metal wire is insulated from coplanar electrodes by a polymer coating.

The static magnetic field is applied using a permanent neodymium magnet. Based on the relation between the splitting between ODMR resonances and the magnetic field magnitude ${ }^{8}$, the magnitude of the magnetic field applied along the [100] is estimated to be $0.50 \mathrm{mT}$ and the amplitude of the magnetic field applied along the [111] direction to be $2.0 \mathrm{mT}$.

For ODMR and PDMR measurements, the microwave frequency is swept in the range $2,800-2,940 \mathrm{MHz}$ with a step of $1 \mathrm{MHz}$. For each measurement, 100-200 spectra are recorded and averaged.

Fitting of ODMR and PDMR spectra. To determine the splitting between mag netic resonances at zero-magnetic field (zero-field splitting, or ZFS), the FWHM and the contrast, PDMR and ODMR spectra recorded in the absence of external magnetic field were fitted to the sum of two Lorentzian functions:

$$
S(F)=S_{0}-\frac{A_{1} g^{2}}{\left(F-F_{0}+A_{\mathrm{sp}}\right)^{2}+g^{2}}-\frac{A_{2} g^{2}}{\left(F-F_{0}-A_{\mathrm{sp}}\right)^{2}+g^{2}},
$$

where $F$ is the microwave frequency, $S_{0}$ is the photocurrent or the photoluminescence intensity outside of resonances, $A_{1}$ and $A_{2}$ the amplitudes of respectively the first and the second magnetic resonances, $F_{0}$ the central frequency, $A_{\text {sp }}$ the ZFS and $2 g$ the FWHM. The contrast presented in Fig. 4 corresponds to the amplitude $A_{2}$ of the second zero-magnetic field resonance 
(situated at $F_{2}=F_{0}+A_{\mathrm{sp}} \sim 2,873 \mathrm{MHz}$ ). Examples of ODMR and PDMR spectra fitting are presented in the Supplementary Fig. 6a,b and the corresponding fitting parameters are presented in the Supplementary Table 1.

Evaluation of the electric field. Although the voltage applied in between coplanar electrodes results in a partially non-uniform electric field, a simple estimation of the magnitude of the applied electric field $F_{\text {app }}$ is obtained by dividing the applied voltage $U$ by the distance $L$ in between electrodes. The magnitude of the local electric field in diamond is calculated using the Lorentz local field approximation ${ }^{45}$. In this approximation, the local electric field $F_{\text {loc }}$ can be expressed as

$$
F_{\text {loc }}=F_{\text {app }} \frac{\left(\varepsilon_{\mathrm{r}}+2\right)}{3},
$$

where $\varepsilon_{\mathrm{r}}$ is the relative electric permittivity of diamond.

\section{References}

1. Maurer, P. C. et al. Room temperature quantum bit memory exceeding one second. Science 336, 1283-1286 (2012).

2. Pfaff, W. et al. Unconditional quantum teleportation between distant solidstate quantum bits. Science 345, 532-535 (2014).

3. Maze, J. R. et al. Nanoscale magnetic sensing with an individual electronic spin in diamond. Nature 455, 644-647 (2008).

4. Häberle, T., Schmid-Lorch, D., Karrai, K., Reinhard, F. \& Wrachtrup, J. Highdynamic-range imaging of nanoscale magnetic fields using optimal control of a single qubit. Phys. Rev. Lett. 111, 170801 (2013).

5. Dolde, F. et al. Nanoscale detection of a single fundamental charge in ambient conditions using the $\mathrm{NV}^{-}$center in diamond. PRL 112, 097603 (2014).

6. Staudache, T. et al. Nuclear magnetic resonance spectroscopy on a (5-Nanometer) ${ }^{3}$ sample volume. Science 339, 561-563 (2013).

7. McGuinness, L. P. et al. Ambient nanoscale sensing with single spins using quantum decoherence. New J. Phys. 15, 073042 (2013).

8. Rondin, L. et al. Magnetometry with nitrogen-vacancy defects in diamond. Rep. Prog. Phys. 77, 056503 (2014).

9. Hadden, J. P. et al. Strongly enhanced photon collection from diamond defect centres under micro-fabricated integrated solid immersion lenses. Appl. Phys. Lett. 97, 241901 (2010).

10. Momenzadeh, S. A. et al. Nanoengineered diamond waveguide as a robust bright platform for nanomagnetometry using shallow nitrogen vacancy centers. Nano Lett. 15, 165-169 (2014).

11. Li, L. et al. Efficient photon collection from a nitrogen vacancy center in a circular bullseye grating. Nano Lett. 15, 1493-1497 (2015).

12. Childress, L. \& Hanson, R. Diamond NV centers for quantum computing and quantum networks. MRS Bull. 38, 134-138 (2013).

13. Wildanger, D., Maze, J. R. \& Hell, S. W. Diffraction unlimited all-optical recording of electron spin resonances. Phys. Rev. Lett. 107, 017601 (2011).

14. Maurer, P. C. et al. Far-field optical imaging and manipulation of individual spins with nanoscale resolution. Nat. Phys. 6, 912-918 (2010).

15. Shields, B. J., Unterreithmeier, Q. P., De Leon, N. P., Park, H. \& Lukin, M. D. Efficient readout of a single spin state in diamond via spin-to-charge conversion. Phys. Rev. Lett. 114, 136402 (2015).

16. Brenneis, A. et al. Ultrafast electronic read-out of diamond NV centers coupled to graphene. Nat. Nanotechnol 10, 135-139 (2014).

17. Liu, K. et al. Simple fabrication scheme for sub-10 nm electrode gaps using electron beam lithography. Appl. Phys. Lett. 80, 865 (2002).

18. Visconti, P. et al. The fabrication of sub- $10 \mathrm{~nm}$ planar electrodes and their use for a molecule-based transistor. Nanotechnology 15, 807 (2004).

19. Bube, R. H. Photoconductivity of Solids (Wiley, 1960).

20. Remes, Z., Petersen, R., Haenen, K., Nesladek, M. \& D’Olieslaeger, M. Mechanism of photoconductivity in intrinsic epitaxial CVD diamond studied by photocurrent spectroscopy and photocurrent decay measurements. Diamond Relat. Mater 14, 556-560 (2005).

21. Simanovskaia, M. et al. Sidebands in optically detected magnetic resonance signals of nitrogen vacancy centers in diamond. Phys. Rev. B 87, 224106 (2013).

22. Jensen, K., Acosta, V. M., Jarmola, A. \& Budker, D. Light narrowing of magnetic resonances in ensembles of nitrogen-vacancy centers in diamond. Phys. Rev. B 87, 014115 (2013).

23. Aslam, N., Waldherr, G., Neumann, P., Jelezko, F. \& Wrachtrup, J. Photo induced ionization dynamics of the nitrogen vacancy defect in diamond investigated by single shot charge state detection. New J. Phys. 15, 013064 (2013).

24. Weber, J. R. et al. Quantum computing with defects. Proc. Natl Acad. Sci. USA 107, 8513-8518 (2010).

25. Deák, P., Aradi, B., Kaviani, M., Frauenheim, T. \& Gali, A. Formation of NV centers in diamond: A theoretical study based on calculated transitions and migration of nitrogen and vacancy related defects. Phys. Rev. B 89, 075202 (2014)

26. Beha, K., Batalov, A., Manson, N. B., Bratschitsh, R. \& Leitenstorfer, A. Optimum photoluminescence excitation and recharging cycle of single nitrogen-vacancy centers in ultrapure diamond. Phys. Rev. Lett. 109, 097404 (2012).

27. Siyushev, P. et al. Optically controlled switching of the charge state of a single nitrogen-vacancy center in diamond at cryogenic temperatures. Phys. Rev. Lett 110, 167402 (2013)

28. Collins, A. T. The Fermi level in diamond. J. Phys. Condens. Matter 14, 3743-3750 (2002).

29. Wotherspoon, A., Steeds, J. W., Catmull, B. \& Butler, J. Photoluminescence and positron annihilation measurements of nitrogen doped CVD diamond. Diamond Relat. Mater 12, 652-657 (2003).

30. Rosa, J. et al. Simultaneous characterization of defect states in CVD diamond by PDS, EPR, Raman and photocurrent spectroscopies. Diamond Relat. Mater 7, 1048-1053 (1998).

31. Heremans, F. J., Fuchs, G.D., Wang, C. F., Hanson, R. \& Awschalom, D. D. Generation and transport of photoexcited electrons in single-crystal diamond. Appl. Phys. Lett. 94, 152102 (2009).

32. Hanson, R., Gywat, O. \& Awschalom, D. D. Room-temperature manipulation and decoherence of a single spin in diamond. Phys. Rev. B 74, 161203 (2006).

33. Tetienne, J. P. et al. Magnetic-field-dependent photodynamics of single NV defects in diamond: an application to qualitative all-optical magnetic imaging New J. Phys. 14, 103033 (2012).

34. Acosta, V. M., Jarmola, A., Bauch, E. \& Budker, D. Optical properties of the nitrogen-vacancy singlet levels in diamond. Phys. Rev. B. 82, 201202 (2010).

35. Dréau, A. et al. Avoiding power broadening in optically detected magnetic resonance of single NV defects for enhanced DC-magnetic field sensitivity. Phys. Rev. B 84, 195204 (2011)

36. Van Oort, E. \& Glasbeek, M. Electric-field-induced modulation of spin echoes of N-V centers in diamond. Chem. Phys. Lett. 168, 529-532 (1990).

37. Dolde, F. et al. Electric-field sensing using single diamond spins. Nat. Phys. 7, 459-463 (2011)

38. Doherty, M. W. et al. Theory of the ground state spin of the $\mathrm{NV}^{-}$center in diamond: I. Fine structure, hyperfine structure, and interactions with electric, magnetic and strain fields. Phys. Rev. B 85, 205203 (2012).

39. Malinauskas, T. et al. Optical evaluation of carrier lifetime and diffusion length in synthetic diamonds. Diamond Relat. Mater 17, 1212-1215 (2008).

40. Scajev, P., Gudelis, V., Ivakin, E. \& Jarasiunas, K. Nonequilibrium carrier dynamics in bulk HPHT diamond at two-photon carrier generation. Phys. Status Solidi A 208, 2067-2072 (2011).

41. Isberg, J. et al. High carrier mobility in single-crystal plasma-deposited diamond. Science 297, 1670-1672 (2002).

42. Jensen, K. et al. Cavity-enhanced room-temperature magnetometry using absorption by nitrogen-vacancy centers in diamond. Phys. Rev. Lett. 112, 160802 (2014).

43. Laraoui, A. \& Meriles, C.A. Rotating frame spin dynamics of a nitrogenvacancy center is a diamond nanocrystal. Phys. Rev. B 84, 161403 (2011)

44. Stepanov, V., Cho, F. H., Abeywardana, C. \& Takahashi, S. High-frequency and high-field optically detected magnetic resonance of nitrogen-vacancy centers in diamond. Appl. Phys. Lett. 106, 063111 (2015).

45. Choy, T. C. Effective Medium Theory: Principles and Applications (Clarendon Press, 1999).

\section{Acknowledgements}

This work was supported by the AFOSR/DARPA QuASAR program, The Research Foundation-Flanders (FWO) (Grant agreement G.088812N), the European Union Seventh Framework Programme (FP7/2007-2013) under the project DIADEMS (grant agreement no. 611143), the Ministry of Science and Education of the Czech Republic (Contract CZ.1.07/2.3.00/20.0306), the German-Israeli Project Cooperation (DIP) program and NSF (Grant No. ECCS-1202258). The authors are grateful to P. Kehayias for helpful discussions, to K. Aulenbacher and the MAMI staff for electron irradiation and to Y. Balasubramaniam, P. Robaeys and M. McDonald for electrodes fabrication.

\section{Author contributions}

E.B., P.S. and M.G. performed the experiments. A.J. prepared the irradiated and annealed diamond samples. J.H. wrote the software used for PDMR measurements. E.B. processed the data. M.N., D.B. and F.J. supervised the work. E.B. and M.N. wrote the manuscript. All authors discussed the results and commented on the manuscript.

\section{Additional information}

Supplementary Information accompanies this paper at http://www.nature.com/ naturecommunications

Competing financial interests: The authors declare no competing financial interests. 
Reprints and permission information is available online at http://npg.nature.com/ reprintsandpermissions/

How to cite this article: Bourgeois, E. et al. Photoelectric detection of electron spin resonance of nitrogen-vacancy centres in diamond. Nat. Commun. 6:8577 doi: 10.1038/ncomms9577 (2015). (c) (i) This work is licensed under a Creative Commons Attribution 4.0 International License. The images or other third party material in this article are included in the article's Creative Commons license, unless indicated otherwise in the credit line; if the material is not included under the Creative Commons license, users will need to obtain permission from the license holder to reproduce the material. To view a copy of this license, visit http://creativecommons.org/licenses/by/4.0/ 